\title{
Title - UltraTrack: Software for semi-automated tracking of muscle fascicles in sequences of B- mode ultrasound images
}

\author{
Authors - Dominic James Farris ${ }^{\mathrm{a}}$ and Glen A. Lichtwark ${ }^{\mathrm{a}}$ \\ a School of Human Movement \& Nutrition Sciences, Level 5, Building 26B, Blair Drive, The \\ University of Queensland, Brisbane, QLD 4072, Australia.
}

Email addresses - d.farris@uq.edu.au,g.lichtwark@uq.edu.au

Corresponding Author - Dominic James Farris, School of Human Movement \& Nutrition Sciences, Level 5, Building 26B, Blair Drive, The University of Queensland, Brisbane, QLD 4072, Australia. Email: d.farris@uq.edu.au, Tel: +61073365 6097.

\begin{abstract}
1Abstract
2Background - Dynamic measurements of human muscle fascicle length from sequences of B-mode 3ultrasound images have become increasingly prevalent in biomedical research. Manual digitisation of these 4images is time consuming and algorithms for automating the process have been developed. Here we 5 present a freely available software implementation of a previously validated algorithm for semi-automated 6tracking of muscle fascicle length in dynamic ultrasound image recordings, "UltraTrack". Methods 7 UltraTrack implements an affine extension to an optic flow algorithm to track movement of the muscle 8 fascicle end-points throughout dynamically recorded sequences of images. The underlying algorithm has 9 been previously described and its reliability tested, but here we present the software implementation with 10features for: tracking multiple fascicles in multiple muscles simultaneously; correcting temporal drift in 11 measurements; manually adjusting tracking results; saving and re-loading of tracking results and loading a 12range of file formats. Results - Two example runs of the software are presented detailing the tracking of 13 fascicles from several lower limb muscles during a squatting and walking activity. Conclusion - We have 14 presented a software implementation of a validated fascicle-tracking algorithm and made the source code 15 and standalone versions freely available for download.
\end{abstract}

16Keywords - fascicle, tracking, ultrasound, automated, affine optic flow, Matlab.

17

\section{Introduction}

19The in-vivo measurement of human skeletal muscle function has long been of interest to scientists and 20engineers from a variety of backgrounds including: physiology, anatomy, evolutionary biology, ergonomics, 21 biomedical engineering and human movement science. Of fundamental importance in this area is the 22 measurement of muscle architecture and how it dynamically changes with muscle contraction. A key 23 feature of muscle architecture is muscle contractile element length and many important research questions 24 can be answered with knowledge of how contractile elements change length during muscle contraction. 25The basic contractile element of muscle is the sarcomere and many sarcomeres in series make up muscle 26 fibres, which in turn are bundled into fascicles. Sarcomeres are microscopic in size and so it is common to 27 measure fibre or fascicle length changes as a proxy.

28 In the last twenty years, B-mode ultrasound has risen in popularity as a means of imaging skeletal muscle in 29 static and dynamic conditions. Cross-sectional ultrasound images of skeletal muscle allow identification of 30 muscle fascicles [1]. By sampling images at a suitable rate, the length changes of muscle fascicles during 31 dynamic contractions have been characterised through digitisation of image sequences $[2,3]$. Initially, the 32 digitisation process was manual, making it extremely time consuming and somewhat subjective. More 33recently, image processing algorithms to partially or fully automate the digitisation process have been 34 developed [4-6]. These employ a variety of methods including Kanade-Lucas-Tomasi feature tracking and 35 affine transformation extensions to optic flow. The validity and reliability of the affine optic flow algorithm 36 of Cronin et al. [4] was published some time ago [4, 7]. The algorithm and the software implementation 37 have since been substantially expanded and in this paper we present the refined algorithm and freely 
38available software package, UltraTrack version 4.1, the source code for which is written in Matlab (The 39MathWorks Inc., USA). A new 'key-frame correction' algorithm has been added to help remove temporal 40drift (accumulation of error with time) in the tracking that had previously limited the length of image 41sequences that could be tracked. A manual correction is also added as an alternative for making 42adjustments to tracking results. New features of the software include: compatibility with more image file 43formats, faster implementation of the affine flow algorithm, tracking of multiple fascicles and regions of 44interest, image auto-cropping function, saving and re-loading of tracking data and a choice of moving or 45 fixed region of interest for the affine flow calculations.

\section{Computational Methods}

\subsection{Overview}

49The software requires an image sequence as input (see 3.1 for details) and the user must manually define 50region(s) of interest and the representative fascicle(s) that they wish to track (see 3.3 for details) in the 51 image frame from which they wish to start tracking. Once region(s) and fascicle(s) are defined, the user can 52 process the image sequence, which implements an algorithm based on the affine optic flow model 53described in section 2.2 to track fascicle length in the subsequent frames of the image sequence. The 54algorithm steps through the image sequence one frame at a time, computing the optic flow between 55 consecutive images and applying the affine transformation to calculate the new position and length of the 56 fascicle(s). Because this is an iterative process, any tracking errors in individual frames may accumulate over 57time and be compounded, causing the fascicle position and length to 'drift' as the algorithm progresses 58 through image sequences. To correct for this, we have developed the 'key-frame correction' method that is 59described in section 2.3 .

\subsection{The affine optic flow model}

61The main algorithm in the software implements an affine optic flow model to compute the optic flow and 62the affine transformation between consecutive images in the sequence. Once the model parameters have 63been computed for a pair of images, the affine transformation matrix can be used to determine the new 64 Cartesian coordinates of any point defined in the first image, in the second image. This process is applied to 65 compute the displacement of the fascicle endpoints from one image to the next. The model and algorithm 66 have been described and their use validated previously [4, 7], but for completeness we will also describe it 67here.

68The model is based upon an affine extension of the well-established Lucas-Kanade method $(2,23)$. The 69affine optic flow model has six parameters: $v_{x t}$ - optic flow at the image origin (top left corner) in the $x$ 70direction; $v_{y t}$ - optic flow at the origin (top left corner) in the y-direction; $d$ - rate of dilation; $r$ - rate of 71rotation; $s_{1}$ - shear along the main image axis; $s_{2}$ - shear along the diagonal image axis. These parameters 72 can be used to estimate the flow vector (change in position vx, vy) at specific points in the image $(x, y)$ by 73applying the following first order model (30):

$74\left(v_{x}, v_{y}\right)=\left[\begin{array}{lll}x & t & 1\end{array}\right] \times\left[\begin{array}{cc}d+s_{1} & s_{2}+r \\ s_{2}-r & d-s_{1} \\ v_{x t} & v_{y t}\end{array}\right]$

75The Matlab algorithm used here (contributed by Dr. David Young and available from Matlab Central - see 76acknowledgments) implements a least squares fit of the above parameters to estimates of the spatial and 77temporal gray-level gradients on a rectilinear grid within the defined region of interest. To calculate the 78spatial and temporal gradients, the images are first smoothed in both the $\mathrm{x}$ and $\mathrm{y}$ components by 79convoluting the image with a 1D Gaussian mask. In the implementation used here, the Matlab function 80 "fspecial" is used to create the mask with a width of 2.6 times the parameter "sigma," which is the standard 81deviation of the distribution. To calculate the spatial gradients, the symmetric local difference of the average 82 of the two smoothed images is calculated by two-dimensional convolution in the $x$ and $y$ directions using a 83 simple linear gradient function. The temporal gradients are calculated as the difference between the two 
84 smoothed images. The affine flow parameters are then solved by a least squares fit of the parameters to 85 achieve the given spatial and temporal gradients. The $\mathrm{x}$ and $\mathrm{y}$ grids along with the spatial and temporal $86 g r a d i e n t s$ are then resampled ( 3 pixels width) to reduce the data used in the least squares solution and 87improve processing time. Only data within the defined region of interest are used to obtain the least 88 squares solution. Once the parameters for the model are determined, the flow at individual points can be 89determined using Eq. 1. Using the model it is possible to calculate the change in position of any $x$-y point 90from one image to the next using the affine transformation. This includes points outside of the region of 91interest and thus, the fascicle endpoints can be outside the region of interest. In our algorithm, the 92calculated affine transformation is applied to the Cartesian coordinates of the defined fascicle end points 93from the first image frame of each pair to calculate the new coordinates in the subsequent image. This 94iterative approach allows fascicle length to be defined for each ultrasound image in a sequence.

\subsection{Key-frame correction algorithm}

960nce the affine optic flow algorithm has been run, the length of the fascicle(s) has been calculated in each 97 frame of the image sequence. It is not uncommon for image quality to be lower at some point during the 98sequence, causing tracking errors in those frames. Because the algorithm is iterative, these errors can 99accumulate or be compounded with each iteration and introduce a low-frequency 'drift' to the tracking 100results. To remove this drift from the signal, we have introduced a post-processing algorithm, the 'keyframe 101correction'.

102The key-frame correction requires that the data be from either a periodic activity or an activity that has 103some recurring reference state, such that the fascicle length can be assumed to be at a similar length at the 104 same fraction of consecutive activity cycles or at each occurrence of the reference. For example, the muscle 105 fascicles should be at similar lengths if the configuration of the joints that the muscle crosses (e.g. knee and $106 a n k l e$ for the gastrocnemius muscle) and muscle forces are similar at two different time points. For instance, 107during steady state walking, the muscle fascicles should be at similar lengths at the same fraction of the gait 108 cycle (e.g. foot contact, toe-off). The user must identify 'key frames' in the image sequence that occur 109 where the fascicles should be considered to be at similar lengths. Once key frames are identified, the key110 frame images are treated as a separate image sequence and the optic flow and affine transformation are 111 computed between each consecutive pair of key-frame images. The first key-frame is assumed to be error 112 free because it should be near the start of the image sequence and therefore the fascicle endpoint 113 coordinates and lengths are not corrected for this frame. For the subsequent key-frames, the coordinates of 114the fascicle endpoints are recomputed by applying the new affine transformation to the coordinates from 115the previous key-frame. The horizontal and vertical displacements between the original endpoint 116coordinates and the new endpoint coordinates are taken to be the magnitudes of the errors in each 117 direction at that point in the full image sequence. The measurement drift between each pair of key-frames 118 is assumed to accumulate linearly with time and the key-frame error values are linearly interpolated to 119 compute horizontal and vertical error values at the time of each image frame between each pair of key120 frames. The error value is subtracted from the fascicle endpoint coordinates to compute the new position of 121each fascicle endpoint in each image frame. These are referred to as corrected fascicle endpoints and are 122 used to compute a corrected fascicle length for each frame (see section 4 for examples).

123The key-frame correction may not always provide satisfactory results. This may be because the assumption 124that errors accumulate linearly is not always appropriate. As noted previously errors are often due to a sub125 sequence of images where tracking is poor, so manually correcting (section 3.5) during such sub-sequences 126 may produce better results than the key-frame correction.

\section{Program Description}

\subsection{Input files and file conversion}

130Version 4.1 of UltraTrack handles any format of input file that is supported by the inbuilt Matlab 131'VideoReader' (post R2010a) or 'mmreader' (pre R2010a) functions for the image sequences as well as 132specifically formatted MAT, B8 and B32 files. B8 and B32 file types are generated by Ultrasonix ultrasound 133systems (BK Ultrasound, USA) and are read using custom Matlab functions. The formats supported by the 
134 'VideoReader' and 'mmreader' functions are platform dependent with the exception of AVI, which is 135supported across platforms and has been most extensively tested with the UltraTrack software. For details 136of other supported file types, visit the support documentation of the MathWorks website 137(www.mathworks.com). It should be noted that the loading of files that require 'VideoReader' or 138 'mmreader' functions is considerably slower than loading a MAT file containing the image sequence.

139To use a MAT file as the input file, it must contain a Matlab structure named 'TVDdata' with the following 140fields: 'Fnum' - a numeric value for the number of frames in the image sequence; 'Width' - a numeric value 141 specifying how many columns of pixels the images consist of; 'Height' - a numeric value specifying how 142 many rows of pixels the images consist of; 'Time' - a column vector of time stamps corresponding to each 143 frame in the image sequence; 'Im' - a three dimensional array where each 'page' is a grey scale image of 144data type uint8 values. The MAT input file content was specifically developed from the conversion of TVD 145files that are generated by EchoWave II ultrasound imaging software (Telemed, Lithuania). With the 146UltraTrack software, we provide a Matlab script (TVD_2_MAT.m) and functions that convert TVD files to 147MAT files with the necessary content. The conversion script interfaces with the latest releases of EchoWave $148 \mathrm{II}$ (Telemed, Lithuania) that must be installed and running when the script is executed. Also, the user must 149 be running Matlab as an administrator for the script to execute.

\subsection{Loading, cropping and cutting the image sequence}

151Loading, cropping and cutting the image sequence are the first three steps in the process of tracking a file. 152Loading is simply a case of selecting 'Open Video File' from the 'File' drop-down menu and you will be 153prompted to select the appropriate file. When the file has finished loading, the first image frame will be 154displayed. In this software, 'cropping' refers to removing any surrounding borders from the image and it is 155particularly important to remove any borders above or below the image. This is because the scale of the 156image (pixels $/ \mathrm{mm}$ ) is calculated by dividing the number of rows of pixels by the specified image depth. The 157image depth refers to the scanning depth in $\mathrm{mm}$ and is input either by the editable text box on the user 158interface or from the 'Settings' drop-down menu. If the user does not wish to crop the image but knows the 159scale factor for the image and the image height (in pixels), an appropriate image depth can be calculated 160 and used. For images produced by EchoWave II software (Telemed, Lithuania), auto crop functionality is 161 available from the 'Image' drop-down menu that will automatically remove all borders from the image.

162 'Cutting' the sequence refers to removing frames from the start or end of the video sequence. It is 163imperative that any cutting of the image sequence is done before any of the steps in the tracking process 164described below are undertaken. The sequence can be cut by navigating to the appropriate frame and 165clicking on the 'Cut Before' or 'Cut After' buttons on the 'Setup Tracking' panel of the user interface. Cutting 166the image sequence down to the necessary number of frames will speed up the tracking proportionally. 167 Cutting or cropping does not affect the original input file in any way.

\subsection{Defining regions of interest and fascicles}

169The affine optic flow computations are applied to a specified region of interest (ROI) within the images. This 170ROI is defined using the 'Define ROI' button on the 'Setup Tracking' panel that allows the user to define the 171vertices of a polygon by clicking on the image before double clicking inside the completed polygon to 172finalise the ROI, which will display as a red dashed outline when finalised. Typically, the ROI is defined as the 173outline of the cross section of the muscle of interest. Our experience has taught us that this should usually 174 include the thick muscle fascia, or aponeurosis, that borders the muscle. However, finding the ROI definition 175that works best for your image sequences may require some trial and error. Whether or not to include 176aponeurosis may depend on whether it can be distinguished separately from that of adjacent muscles and 177any relative movement that occurs between muscles (or other tissues).

178 In version 4.1 of UltraTrack, it is now possible to have multiple ROI's such that more than one muscle can be 179 concurrently tracked. ROI's can be added by clicking on the add button next to the drop-down list of region 180 numbers. The number displayed on the region number list is that of the currently selected region. The user 181has the option to select a fixed ROI by checking the 'Fixed ROI' check box. This means that the ROI will 182remain the same throughout the image sequence as the algorithm steps though the images. If this box is 183 not checked, then the ROI vertices have the affine flow transformation applied to them in the same way as 184the fascicle endpoints and the ROI will move and change shape to account for movement of the muscle. 
185This latter option is only recommended if there is significant vertical displacement of the muscle during the 186sequence. It will also tend to reduce the width of the ROI as the sequence progresses, which results in the 187 tracking region changing across a video sequence and therefore may influence the tracking results.

188 Within each ROI, at least one fascicle must be defined. To define a fascicle, click on the 'Define Fascicle' 189 button and you will be able to click on or around the image to define the endpoints of the fascicle. It is 190possible to define multiple fascicles within a ROI and fascicles can be added to the currently selected ROI by 191clicking on the add button next to the drop-down list of fascicle numbers. Fascicle numbering starts from 192one for each ROI. For example, if you wish to track two ROI's with one fascicle in each, the ROI list should 193have the numbers one and two available and the fascicle list has only the number one. In this case, which 194 fascicle is selected depends only on which region is selected. Regions and fascicles should be defined in the 195image from which you wish to start the tracking and this should be the first frame of the sequence. 196Therefore users should cut the image sequence so that it starts from the desired start frame.

\subsection{Running the tracking algorithm}

198 Having defined the appropriate ROI(s) and fascicle(s) the user can then run the affine flow algorithm by 199clicking the 'Process All' button on the user interface or selecting that option from the 'Tracking' menu. This 200uses the affine optic flow model described in section 2.2 to track the defined fascicles throughout the image 201sequence. Once the algorithm has finished, fascicle lengths will be plotted in the axes at the top right of the 202user interface and the tracked fascicle(s) and ROI(s) will be overlaid on the image sequence. A more manual 203approach to tracking can be performed by clicking through the sequence frame-by-frame rather than 204selecting "process all".

\subsection{Making manual adjustments}

206It may be desirable to make adjustments to the tracking results if there are frames in which the tracking 207algorithm has been unable to suitably track the fascicle. Potential causes of poor tracking are: large 208 movements between frames (often caused by insufficient sampling rates) and large changes in image 209brightness caused by movement not in the plane of the image (constant image brightness is a key 210 assumption behind optic flow calculations). As described elsewhere in this paper, a key-frame correction has 211 been developed to remove drift in recordings of periodic activities or activities with a common set point 212(e.g. a muscle that is fully relaxed before and after a contraction). However, there may be occasions for 213 which it is more appropriate simply to make a manual correction in certain frames. For this purpose there is 214the manual correction panel on the user interface that allows the user to adjust the position of the upper ( $U$ 215button) or lower (L button) endpoint of the fascicle. Upper or lower refers to the vertical position within the 216image of that fascicle endpoint. Having clicked on the $U$ or $L$ button, the user can then use the cursor to 217 select a new position for the fascicle endpoint and the affine transformation will be re-applied to the new 218 coordinates and all subsequent frames to account for the adjustment. Corrected fascicle lengths will be 219 calculated and displayed in the fascicle length plot and the corrected fascicle overlay will be displayed on 220the image sequence in yellow.

\subsection{Making a key-frame correction}

222The key-frame correction and its purpose have been described in detail in section 2.3. It can only be 223performed once the affine optic flow algorithm has been run and can be run on data that has had manual or 224 previous key-frame corrections made to it (although the latter is not recommended). To make a correction, 225the user must first create a list of key-frames by navigating to each key-frame using the video controls and 226 clicking 'Add' button on the key-frame correction panel. The current frame number should be added to the 227 list of key-frames. To remove a key-frame from the list, the user simply selects that frame in the list and 228 clicks the 'Delete' button underneath the list. When the key-frames have been selected, the user can choose 229 what ROI and fascicle to apply the correction to using the drop-down lists. Most often the user will simply 230select 'all' and correct all ROI's and fascicles. Having set these selections, the user can click the 'Run Key231 frame Correction' button and the correction algorithm described in section 2.3 will be applied. If the results 232of the correction are unsatisfactory, the user can click the 'Undo Correction' button and the tracking will be 233 reset to its previous state. If more than one correction has been applied, the user can only undo the most

234recent correction. 


\subsection{Saving tracking results}

236Two options exist for saving tracking results to file: MAT file and TXT file. MAT file is recommended as this 237saves all the necessary information to reload the tracking results along with the image sequence if 238necessary. Saving to a TXT file will only save the fascicle length and pennation angle data. Tracking data can 239be saved using the 'Save Tracking Data' or 'Save Tracking Data as...' options from the 'File' menu. When 240saved to a MAT file, the 1-D structure 'Fdat' is saved in the file. Fdat contains the field 'Region' which is a $2411 x n$ structure where $n$ is the number of ROI's. The Region structure contains the fields: 'FL' - fascicle length; 242 'PEN' - pennation angle and 'Time' - time stamps corresponding to each of the data points in FL and PEN. FL 243and PEN are $m \times n$ 2-D arrays where $m$ is the number of tracked image frames and $n$ is the number of 244 fascicles defined for that ROI. It should be noted that pennation angle is not a true pennation angle and is in 245 fact the angle of the fascicle relative to the image horizontal axis. There is also an option in the Tracking >> 246Fascicle Tracking menu to 'Save Fascicle' that saves the tracking data for current frame to a MAT file.

\subsection{Loading tracking data}

248If tracking results were saved to a MAT file, they can be reloaded after the image sequence has been loaded 249 with the 'Load All Tracked Frames' option in the 'Fascicle Tracking' sub-menu of the 'Tracking' menu. Doing 250 so will cut the image sequence to the number of previously tracked frames. If a single frame of data has 251 been saved with 'Save Fascicle' then it can be reloaded as the current frame's data using Tracking >> Fascicle 252Tracking >> Load Fascicle.

253

\section{Sample Runs}

\subsection{Gastrocnemius and soleus fascicles during squatting}

$256 \mathrm{As}$ an example run of the software we present the tracking of a gastrocnemius fascicle and a soleus muscle 257 fascicle during a repeated squatting movement. B-mode ultrasound imaging was performed using a 96258element linear array ultrasound transducer (LV7.5/60/96Z, Telemed, Lithuania) operating at a central 259 frequency of $6 \mathrm{MHz}$, a depth of $65 \mathrm{~mm}$ and sampling images at 80 frames per second. The participant was a 260 healthy young male athlete and he performed two repetitions of a squatting movement (for details see [8]). 261The image sequence was recorded using EchoWave II software as a TVD file and converted to MAT file using 262our custom TVD_2_MAT script described in section 3.1. The sequence was loaded into UltraTrack 4.1 and 263auto-cropped to remove image borders before the sequence was cut down to the relevant frames for 264 analysis (using 'cut before' and 'cut after' buttons and synchronously collected ground reaction force data). $265 \mathrm{We}$ then defined the first ROI (surrounding the gastrocnemius muscle) in the first frame by selecting 'Define 266ROI' button and defining a polygon around the portion of the image where gastrocnemius was visualised 267(Figure 1). Here, we used a fixed ROI by checking the 'Fixed ROI' check box (Figure 1). We then selected the 268 'Define Fascicle' button and clicked on the endpoints of a clearly visible gastrocnemius fascicle in the centre 269 of the ROI in the first image (Figure 1). A second ROI was added by selecting the '+' button next to the ROI 270 drop-down list. The second ROI was then made the current ROI by selecting ' 2 ' from the ROI drop-down list 271 and subsequently defined by clicking 'Define ROI' and outlining a polygon surrounding the soleus muscle. 272The soleus fascicle was then defined (Figure 1) using 'Define Fascicle'. Note that the fascicle count restarts 273 for each region, so you do not need to add a fascicle to the drop-down list. We then clicked the 'Process All' 274 button to run the affine optic flow algorithm.

275The fascicle length data output from the affine optic flow algorithm are shown in Figure 2A. As one might 276predict, the muscle fascicles of both muscles lengthened during the downward phase of the squat motions 277 and shortened during the upward phase. The length change was more pronounced in soleus than lateral 278 gastrocnemius due to soleus spanning only the ankle joint whereas gastrocnemius spans the ankle and knee 279joints. The presence of low-frequency drift is evidenced by the progressive drift to longer lengths, but with a 280constant pattern of length changes in each squat. We therefore opted to run a key-frame correction on this 281data. Using synchronously collected ground reaction force data, we identified the times in between squats 282when the participant was standing still and selected the corresponding image frames as key-frames using 283the video controls and 'Add' button on the Key-frame Correction panel (Figure 1). We then clicked the 'Run 284 Key-frame Correction' button and the resulting corrected fascicle length plot is shown in Figure 2B. A video 
285of the image sequence with the tracked fascicles and ROls overlaid was exported using the 'File >> Save 286Video' menu item and is provided in the electronic supplementary information of this manuscript. Further 287 examples will be provided via the software web repository (see section 6).

288The image sequence for this example was $9.2 \mathrm{~s}$ long, with 801 grey-scale image frames of dimensions $645 \mathrm{x}$ 289745 pixels. Stored as a Matlab data file, the file size was 336 MB. UltraTrack was running on a MacBook Pro 290with a $2.5 \mathrm{GHz}$ Intel core i5 processor, 8 GB of RAM and Mac OS X 10.9.5 (Mavericks). The Matlab profiler 291indicated that loading the file took a total time of $17.1 \mathrm{~s}$ and processing with the affine flow algorithm took 292110.3 s.

\subsection{Fascicles in the superficial and deep compartments of tibialis anterior during walking}

294For a second example we present data for the bipennate tibialis anterior (TA) muscle from one young 295healthy individual as they walked at $4.5 \mathrm{kmph}$ on an instrumented treadmill (AMTI, USA). The same 296ultrasound system as in 4.1 was used with the same settings, but positioned on the anterior leg to image TA. 297 We followed the exact same process for tracking as described in 4.1 and the defined fascicles and ROI's are 298shown in Figure 3. The fascicles extended outside the field of view of the transducer and thus, the 299endpoints had to be extrapolated outside the image. Despite this, the algorithm uses information from 300 within the ROI only. That is to say that the affine transformation matrix is computed from the optic flow 301 calculated within the ROI, but applied to the coordinates of the endpoints that may be outside the ROI. The 302initial tracking results are shown in Figure $4 a$ and drift is clearly present, especially for the superficial 303 compartment. Using synchronously collected ground reaction force data we identified the timing of the first 304 active peak in vertical force in each step led with the right foot and used the corresponding frames as key305 frames in a key-frame correction applied to both fascicles. The corrected lengths are shown in Figure $4 \mathrm{~b}$ and 306 a video of the tracking is available as supplementary material.

307The second example was a $10.55 \mathrm{~s}$ clip, with 844 grey-scale image frames of dimensions 645 x 583 pixels. 3085tored as a Matlab data file, the file size was $270 \mathrm{MB}$. UltraTrack was running on the same computer 309described above. The Matlab profiler recorded that file loading took $14.9 \mathrm{~s}$ and processing with the affine 310 flow algorithm took 97.7s. It should be noted that loading an AVI file as opposed to a MAT file takes 311 significantly longer. As an example, a similar an AVI with only 441 frames, but similar dimensions took $96.3 \mathrm{~s}$ 312 to load.

\section{Hardware / software specifications}

\subsection{Standalone version}

316A standalone application installer has been created for MacOS and Windows platforms. The standalone 317application requires Matlab Runtime 8.5 to be installed and the installer for this is packaged with the 318application installer.

\subsection{Source Code}

320For users wishing to run UltraTrack 4.1 using the source code, the Matlab M and FIG files required are 321 available. Relevant folders must be added to the Matlab path and the in-built image processing toolbox is 322required. UltraTrack v4.1 has been tested in Matlab R2015a. There are known graphics problems running 323 UltraTrack 4.1 with R2014a and b but prior releases have no such issues. This is likely related to problems 324 with updates to Matlab graphics objects in R2014a. Separate versions are available for MacOS and Windows 325 platforms, but the only differences are cosmetic - the source code is essentially the same.

\subsection{File conversion}

327M files are provided with the software for the conversion of TVD files (created by EchoWave II software, 328Telemed, Lithuania) to MAT files. A beta version of EchoWave II (freely available for download from 329 pcultrasound.com) must be installed and running when executing these files in Matlab, which must be run 330 as an administrator.

\subsection{System Requirements}


332The requirements for installing and running Matlab Runtime 8.5 and Matlab 2015a are: Windows 333Windows XP or later; 3-4 GB of disk space (typical installation), 2 GB of RAM. Mac - Mac OS X 10.9.5 334(Mavericks) or later, 3-4 GB of disk space (typical installation), 2 GB of RAM.

335

3366. Mode of availability

337 ltraTrack 4.1 is available free of charge for non-commercial use from the 'files' page of the website 338(https://sites.google.com/site/ultratracksoftware/file-cabinet). The website has additional materials and 339 examples.

340

\section{Acknowledgements}

The authors would like to acknowledge Dr David Young of The University of Southampton (UK) who wrote the Matlab classes for implementing the affine optic flow algorithm.

\section{References}

[1] T. Fukunaga, Y. Kawakami, S. Kuno, K. Funato, S. Fukashiro, Muscle architecture and function in humans, J. Biomech., 30 (1997) 457-463.

[2] T. Fukunaga, Y. Ichinose, M. Ito, Y. Kawakami, S. Fukashiro, Determination of fascicle length and pennation in a contracting human muscle in vivo, J. Appl. Physiol., 82 (1997) 354-358.

[3] G.A. Lichtwark, K. Bougoulias, A.M. Wilson, Muscle fascicle and series elastic element length changes along the length of the human gastrocnemius during walking and running, J. Biomech., 40 (2007) 157 164.

[4] N.J. Cronin, C.P. Carty, R.S. Barrett, G. Lichtwark, Automatic tracking of medial gastrocnemius fascicle length during human locomotion, J. Appl. Physiol., 111 (2011) 1491-1496.

[5] J. Darby, E.F. Hodson-Tole, N. Costen, I.D. Loram, Automated regional analysis of B-mode ultrasound images of skeletal muscle movement, J. Appl. Physiol., 112 (2012) 313-327.

[6] G.-Q. Zhou, P. Chan, Y.-P. Zheng, Automatic measurement of pennation angle and fascicle length of gastrocnemius muscles using real-time ultrasound imaging, Ultrasonics, 57 (2015) 72-83.

[7] J.G. Gillett, R.S. Barrett, G.A. Lichtwark, Reliability and accuracy of an automated tracking algorithm to measure controlled passive and active muscle fascicle length changes from ultrasound, Computer Methods in Biomechanics and Biomedical Engineering, 16 (2013) 678-687.

[8] D.J. Farris, G.A. Lichtwark, N.A. Brown, A.G. Cresswell, Deconstructing the power resistance relationship for squats: A joint-level, Scandinavian Journal of Medicine \& Science in Sports, (2015) T aheadofprint. 


\section{Figures Captions}

Figure 1. Snapshot of the UltraTrack GUI after the image sequence has been loaded and an ROI and fascicle have been defined (note: colours have been adjusted for grey-scale display).

Figure 2. Lateral gastrocnemius (black) and soleus (grey) muscle fascicle length over two consecutive squats before (A) and after (B) the key-frame correction was used to remove drift in the signal. The timings of key-frames (which correspond to quiet standing between squats) are highlighted with circles (B). A characteristic lengthening and shortening of the fascicles of both muscles was observed as the participant flexed and then extended their lower limb.

Figure 3. A B-mode ultrasound image of tibialis anterior from the sample run described in section 4.2. Fascicles and ROI's for the superficial and deep compartments of tibialis anterior were defined as shown. Note that the fascicles extend beyond the field of view of the image and thus are extrapolated to where they would insert on the muscle fascia/aponeurosis. Only image data within the ROI's is used to track the extrapolated fascicle endpoints.

Figure 4. Tracking results for fascicles from the superficial (black) and deep (grey) compartments of the tibialis anterior during walking on a treadmill before (A) and after (B) key-frame correction (timings of key-frames indicated with circles in panel B). A video showing the tracking overlaid on the image sequence is available as supplementary material. 


\section{Highlights}

- We have developed a freely available software package for semi-automated tracking of muscle fascicles in B-mode ultrasound image sequences

- Includes features to track multiple fascicles in multiple regions of the image and to correct for measurement drift with time 


\section{File(s) excluded from PDF}

The following file(s) will not be converted:

ESM_1_SquattingPF.gif

ESM_2_WalkingTA.gif

Figure1_BW.eps

Figure2_BW.eps

Figure3_BW.eps

Figure4_BW.eps

Please click 'Download zip file' to download the most recent files related to this submission. 